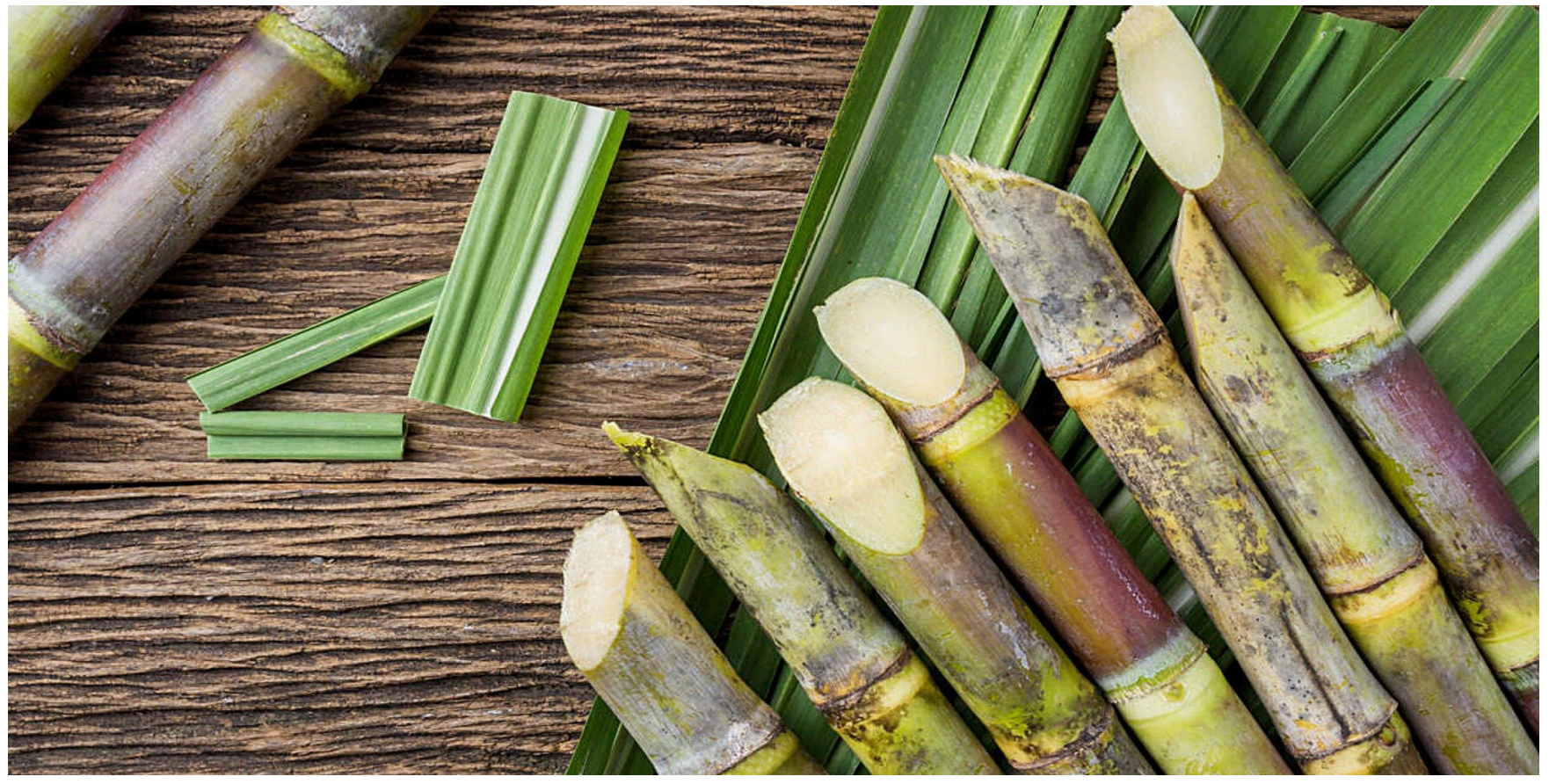

\title{
Análisis comparativo de resultados en el uso de la ceniza de bagazo de caña de azúcar como material sustituyente del cemento portland en el concreto
}

Comparative analysis of results in the use of sugar cane bagasse ash as a substitute material for portland cement in the concrete

Andrés Felipe Ruiz

Tecnólogo en Obras Civiles, andresfeliperj@ufps.edu.co, estudiante de noveno semestre de ingeniería civil de la universidad Francisco de Paula Santander, Cúcuta, Colombia

\section{Carlos Javier Peñaranda}

Tecnólogo en Obras Civiles, carlosjaviermcp@gmail.com, estudiante de noveno semestre de ingeniería civil de la universidad Francisco de Paula Santander, Cúcuta, Colombia
Graciela Fuentes

Tecnóloga en Obras Civiles, gracielafg@ufps.edu.co, estudiante de noveno semestre de ingeniería civil de la universidad Francisco de Paula Santander, Cúcuta, Colombia

María Daniela Semprun

Tecnóloga en Obras Civiles, danielasemprun-97@hotmail.com, estudiante de noveno semestre de ingeniería civil de la universidad Francisco de Paula Santander, Cúcuta, Colombia 


\section{Resumen}

El presente artículo describe la comparación de resultados de diferentes investigaciones que se han realizado con el uso de la ceniza de bagazo de caña de azúcar como sustituyente del cemento portland en la elaboración del concreto estructural, teniendo como objetivos analizar la actividad puzolánica de las distintas muestras que se tomaron para realizar este articulo y comparar estos resultados con otras investigacioneselaboradasconotrosmateriales. Teniendo en cuenta aspectos importantes como la contaminación ambiental que genera este residuo, sus cantidades de sílice (SiO2) y aluminia (Al2O3) y su proceso de obtención de la caña de azúcar. Como conclusiones se puede observar el compromiso que las empresas que generan este residuo tienen con el medio ambiente desarrollando planes que permitan mitigar el impacto que este produce y también como algunos estudios analizados no recomiendan el uso de este sedimento en la elaboración de concreto estructural debido a su disminuida resistencia a la compresión y a su vez, por el contrario, como otros estudios si lo recomiendan como sustituto parcial de agregado fino, en la producción de mortero.

Palabras clave: Análisis, comparación, contaminación ambiental, producción, riesgos, partículas de polvo, concreto estructural.

\section{Abstract}

This article describes the comparison of results of different investigations that have been carried out with the use of sugarcane bagasse ash as a substitute for Portland cement in the elaboration of structural concrete, with the objective of analyzing the pozzolanic activity of the different samples. that is taken to make this article and compare these results with other investigations made with other materials. Taking into account important aspects such as the environmental pollution generated by this residue, its quantities of silica (SiO2) and aluminum (Al2O3) and its process for obtaining sugar cane. As conclusions you can see the commitment that the companies that generate this residue have with the environment, developing plans that mitigate the impact that this produces and also, as some studies analyzed, do not recommend the use of this sediment in the elaboration of structural concrete due to its reduced resistance. to compression and in turn, on the contrary, as other studies do recommend it as a partial substitute for fine aggregate, in the production of mortar.

Keywords: analysis, comparison, environmental contamination, production, risks, dust particles, structural concrete. 
Análisis comparativo de resultados en el uso de la ceniza de bagazo de caña de azúcar como material sustituyente del cemento portland en el concreto

\section{0}

Introducción

La industria azucarera genera anualmente una gran cantidad de residuos, entre estos se encuentra la denominada ceniza de bagazo de caña (CBC) que resulta de la combustión de este subproducto y cuya dispersión en el ambiente contamina el aire y afecta la salud humana [1]. Actualmente, Brasil es el mayor productor de caña de azúcar en el mundo, donde según una encuesta realizada del 2014 al 2015 el estimado nacional de producción de caña era de 671.69 millones de toneladas. Sin embargo, durante el proceso de extracción, el bagazo de caña de azúcar se genera como un residuo, el cual se utiliza en un $95 \%$ como combustible para generar electricidad en calderas de vapor, proceso que genera la ceniza que se necesita para el aditivo [2].

En Colombia, más específicamente en la región del Valle del Cauca, hay trece empresas que satisfacen la demanda nacional de azúcar, pero no solucionan la problemática ambiental que generan con sus residuos, por lo que se hace necesario buscar alternativas [3]. En varios estudios se ha demostrado que la CBC presenta un elevado contenido de sílice (SiO2) y alúmina (Al2O3), que le dan una buena actividad puzolánica como sustituto parcial del cemento portland y constituye una valiosa alternativa que representa un doble beneficio, por un lado, valoriza un desecho y por otro, contribuye a la reducción de gases de efecto invernadero liberados durante la manufactura de cemento (0.85-1 kg CO2/kg cemento) [4].

Esta es una propuesta interesante, si se considera la enorme proporción de contaminación generada por la industria cementera, que según datos del International Cement Review, su producción mundial para el 2010 alcanzó los 3.3 mil millones de toneladas [5]. En el presente estudio se busca analizar los resultados de varias investigaciones que se han realizado con muestras de ceniza de bagazo de caña de azúcar para comprobar su efectividad como potencial sustituto parcial del cemento Portland que se utiliza para el concreto estructural y así comprobar cuál de todas estas investigaciones arroja los mejores resultados.

Por otro lado, para establecer una posible estimación de los residuos que se generan a través del bagazo de la caña de azúcar, se prevé que por cada tonelada de procesado se producen entre 270 y $290 \mathrm{Kg}$ de bagazo y cada tonelada de este genera $23.9 \mathrm{Kg}$ de ceniza [6]. Para hacer una relación, actualmente los residuos agrícolas como la ceniza de la cascarilla de arroz (la cual contiene una gran cantidad de sílice) han sido utilizados para mejorar las propiedades mecánicas en hormigones y morteros trayendo innumerables ventajas desde el punto de vista ambiental puesto que se le da una reutilización a este residuo [7].

Además, la producción de cemento es uno de los mayores responsables de las emisiones globales de $\mathrm{CO} 2$, principalmente debido a la calcinación de la piedra caliza y la quema de combustibles fósiles, convirtiéndolo así es una de las industrias de carbono más activas [8].

\section{Fundamentación Teórica}

La caña se procesa principalmente para fabricar alcohol y azúcar, esta planta presenta un contenido de sacarosa que oscila entre el 11 y el $16 \%$. (Augstburger et al., 2000) Durante el procesamiento de la caña se pueden adquirir diferentes productos como bebidas refrescantes, materias primas, energía alternativa, alimento para ganado, abonos orgánicos, bebidas alcohólicas, productos alimenticios y biocombustibles [9]. A nivel internacional el país que más produce y exporta azúcar es Brasil con un $20 \%$ seguido por India, Tailandia y China que junto a otros 
países asiáticos conforman el $40 \%$ de la producción mundial. [10].

Las condiciones ambientales en la región del Valle del Cauca hacen posible cultivar esta planta durante los 365 días del año, cuya situación ha permitido a 13 grandes empresas establecerse en esta zona para contribuir a la producción colombiana, aunque estas ayudan a satisfacer el consumo de azúcar a nivel nacional, no mitigan la problemática ambiental, la cual se encuentra alterada por la elaboración del cemento, pues este requiere un alto gasto energético de combustibles y produce una gran emisión de contaminantes por las descarbonatación de la piedra caliza [11].

El residuo de la ceniza de bagazo de caña (CBC) se obtiene del fondo de la caldera y como cenizas volantes, este material es considerado puzolánico por algunos investigadores por presentar en su composición gran cantidad de sílice [12]. De acuerdo con información suministrada por algunos ingenios del Valle, actualmente esta ceniza no es utilizada en la fabricación de productos que beneficien directamente a la comunidad vallecaucana que es afectada por este tipo de cultivo que altera el ecosistema, además de influir en problemas respiratorios. Adicionalmente, este residuo azucarero está siendo almacenado a cielo abierto, y sus partículas finas permiten que el viento las desplace por el aire al igual que por la intervención del agua lluvia [13].

Teniendo en cuenta lo anterior, la presente investigación se enfoca en realizar un análisis comparativo entre estudios prácticos que determinen las propiedades físicas del concreto utilizando la ceniza de bagazo de caña como sustituyente del cemento. Dicha comparación busca determinar la viabilidad de la reutilización de este residuo en la elaboración del concreto estructural, la cual implica una solución factible a los problemas ambientales y sociales anteriormente mencionados.

\section{Materiales y métodos}

En el trabajo se emplea un tipo de investigación cuantitativa, pues se busca una comparación de resultados por medio de herramientas informáticas, estadísticas y matemáticas de diferentes investigaciones que se han desarrollado con la ceniza del bagazo de caña de azúcar como material sustituyente para la mezcla del concreto estructural, permitiendo así ordenar y sistematizar las inquietudes, elaborar de manera organizada los conocimientos, proponer teorías, revisar conceptos y evaluar la información según los diferentes criterios, cuantificando el problema y considerando hipótesis que permitan establecer con mayor facilidad la generalización de los resultados [14].

La finalidad de la investigación de tipo cuantitativa, es comprender y analizar los métodos y técnicas que intentan aproximarse al conocimiento de la realidad social a través de la extensión, alcance y significado de los hechos [15] es aquí donde por medio de esta se buscará organizar y cuantificar los resultados de las investigaciones realizadas con la ceniza de bagazo de caña de azúcar. Las investigaciones objeto de estudio fueron las desrarrolladas por [4-5].

Para la elaboración del instrumento de recolección de información se llevó a cabo una separación de los datos y un análisis comparativo entre estas dos investigaciones, organizando los resultados de estas que pudieran servir para llevar a cabo los objetivos de este artículo. De igual forma, se efectuó un estudio detallado del proceso de realización de estas para comprender de una mejor manera el comportamiento de las muestras de ceniza de bagazo de caña de azúcar que se tomaron en cuenta y en este sentido verificar 
Análisis comparativo de resultados en el uso de la ceniza de bagazo de caña de azúcar como material sustituyente del cemento portland en el concreto

\section{2}

si realmente este residuo puede reutilizarse como sustituyente parcial del cemento portland en la elaboración del concreto estructural.

\section{Resultados}

Conocer un poco sobre el proceso que realizan las empresas productoras de azúcar en Cali, Valle del Cauca y sobre como utilizan la caña de azúcar para obtener este producto del que a su vez se deriva la ceniza del bagazo de caña de azúcar una vez procesada, material que es usado para realizar las investigaciones pertinentes de comparación en este artículo. Se presentan los resultados de dicha comparación entre [4-5] y así mismo evaluar el potencial que tiene este material para sustituir al cemento en la fabricación del concreto o si por el contrario sus propiedades pulozonicas no son adecuadas para este proceso.

Proceso de Obtención de la Ceniza de Bagazo de Caña de Azúcar

El cultivo de caña de azúcar empieza con trabajos para la adecuación del campo donde se va a cultivar, entre los cuales se encuentran nivelación y construcción de obras que se realizan para facilitar el flujo del agua a través de este. Posteriormente se realiza la preparación del suelo para proporcionar un ambiente adecuado para sembrar las semillas y luego se procede a regarlas durante 15 días. El proceso de la cosecha comprende las labores de precosecha, aplicación de madurantes, quema, corte, alce y transporte, las cuales se realizan bajo los parámetros exigidos por la legislación ambiental que las rige y con el menor impacto posible a las comunidades [16].

Así pues, se continua con el proceso de fabricación de la azúcar donde se inicia con el proceso de muestreo de la caña que vienen del campo, para medir su calidad y con esto estimar la cantidad de azúcar que se puede obtener.
Luego, se prosigue con el proceso de molienda en el cual se fracciona la caña para romper su corteza y facilitar la extracción del jugo en los molinos. Al material que sale de cada molino se le adiciona jugo de molino inmediatamente siguiente, para lograr extraer la mayor cantidad de sacarosa de azúcar. Antes de que el bagazo pase por el ultimo molino, se le adiciona agua, con el propósito de mejorar la extracción de jugo y sacarosa. El jugo extraído se hace pasar por sistemas de filtración para separar la mayor cantidad de bagacillo del jugo [17].

Finalmente, en el ultimo molino se obtiene un material llamado bagazo el cual contiene bajo contenido de sacarosa y humedad. Aproximadamente el $10 \%$ de este es vendido para la producción de papel y el restante es enviado a las calderas para ser utilizado como combustible en la generación de vapor, proceso en el cual durante su quema se produce la ceniza del bagazo de caña de azúcar, material utilizado para realizar las investigaciones [1819].

Comparación de resultados

Ceniza de bagazo de caña de azúcar en la resistencia a la compresión del concreto, Universidad César Vallejo de Perú.

En esta investigación se evalúa el efecto de la ceniza de caña de azúcar (CBCA) en la resistencia del concreto de $210 \mathrm{~kg} / \mathrm{cm} 2$, reemplazando parcialmente CBCA por el cemento, en proporciones de 20 y $40 \%$.

Los resultados mostraron que la resistencia a la compresión del concreto disminuyó con el incremento de la proporción de ceniza, por debajo de la mezcla de diseño.

El concreto con $20 \%$ de CBCA tuvo mejor comportamiento a compresión a los 7 y 28 días de curado. La presente investigación fue de 
tipo experimental con posprueba únicamente y grupo control, para lo cual se utilizaron 24 probetas cilíndricas de concreto de $150 \times 300$ $\mathrm{mm}$ distribuidas en un grupo control y dos grupos experimentales, a 7 y 28 días de curado con 4 ensayos cada uno.

Las figuras 1 y 2 presentan la granulometría obtenida en los ensayos realizados al agregado fino y grueso, respectivamente.

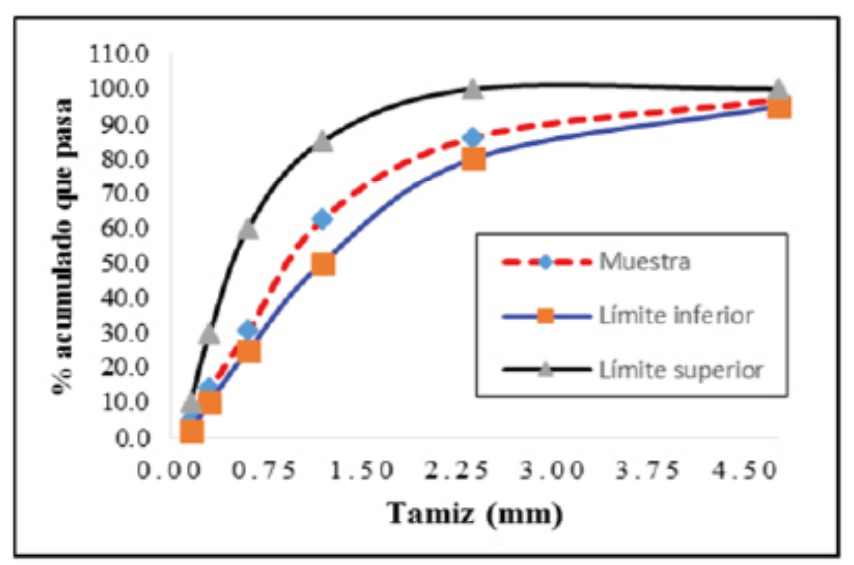

Fuente: Ceniza de bagazo de caña de azúcar en la resistencia a la compresión del concreto, Universidad César Vallejo de Perú

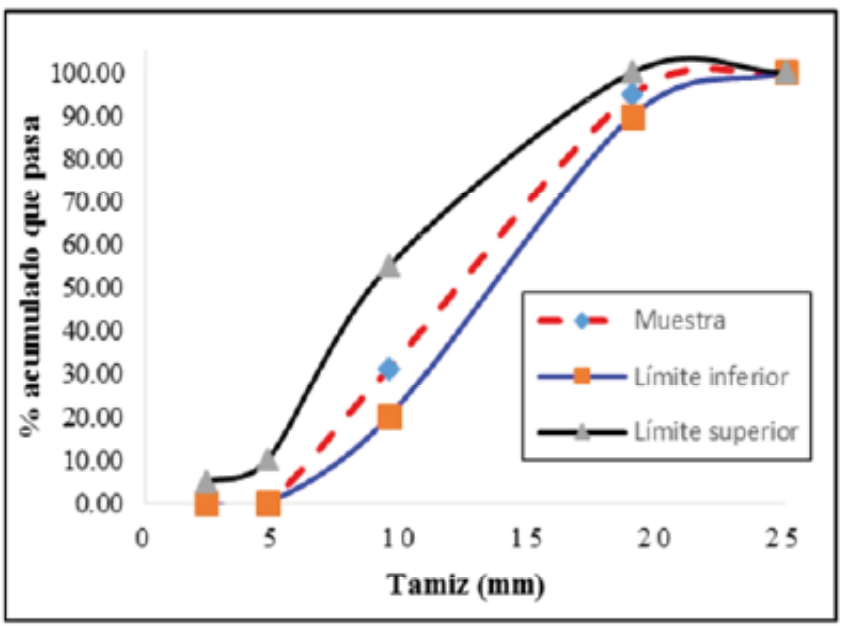

Fuente: Ceniza de bagazo de caña de azúcar en la resistencia a la compresión del concreto, Universidad César Vallejo de Perú.

Los resultados de la primera investigación en principio demuestran que los desechos de caña de azúcar poseen una caracterización que contribuye como material puzolánico para la construcción, de esta manera, se tiene que la CBCA calcinada a una temperatura entre los 800 y 1000 grados Celsius obtiene propiedades que favorecen su actividad puzolánica, de este modo también se analizó el comportamiento de esta actividad dentro de la participación porcentual de la mezcla de fabricación del concreto y se determinó que se debe usar como reemplazo de los constituyentes inertes del cemento en lugar de solo usarlo como aditivo

En las tablas 1 y 2 se pueden observar las proporciones del material que componen las mezlas de concreto estándar

Tabla 1. Proporciones del material que componen la mezcla de concreto estándar.

\begin{tabular}{|c|c|c|}
\hline Material & $\begin{array}{c}\text { Proporción en } \\
\text { peso }\end{array}$ & $\begin{array}{c}\text { Proporción en } \\
\text { volumen }\end{array}$ \\
\hline Cemento & 1 & 1 \\
\hline Agregado fino & 2.29 & 2.21 \\
\hline Agregado grueso & 2.63 & 2.71 \\
\hline Agua & 0.66 & 0.99 \\
\hline \multicolumn{3}{|c|}{$\begin{array}{l}\text { Fuente: Ceniza de bagazo de caña de azúcar en la resistencia a la } \\
\text { compresión del concreto, Universidad César Vallejo de Perú. } \\
\text { abla 2. Proporciones del material que componen la mezcla de f'c= } 210 \mathrm{~kg} \\
\qquad \mathrm{~cm} 2 \text { con } 20 \% \text { y } 40 \% \text { de CBC. }\end{array}$} \\
\hline \multirow{2}{*}{ Material } & \multicolumn{2}{|c|}{ Proporción en peso } \\
\hline & $20 \%$ & $40 \%$ \\
\hline Cemento & 0.8 & 0.6 \\
\hline Ceniza & 0.2 & 0.4 \\
\hline Agregado fino & 2.21 & 2.21 \\
\hline Agregado grueso & 2.71 & 2.71 \\
\hline Agua & 0.99 & 0.99 \\
\hline
\end{tabular}

Fuente: Ceniza de bagazo de caña de azúcar en la resistencia a la compresión del concreto, Universidad César Vallejo de Perú. 
Análisis comparativo de resultados en el uso de la ceniza de bagazo de caña de azúcar como material sustituyente del cemento portland en el concreto

\section{4}

Como se observa en la tabla 3 , en el ensayo de espectrometría de la ceniza se determinó que los componentes químicos de Silicio, aluminio y hierro superan a sus correspondientes para el cemento y por el contrario el porcentaje de calcio no alcanzo los requerimientos necesarios.

Tabla 3. Composición química inorgánica elemental de las cenizas de bagazo de caña de azúcar (CBCA)

\begin{tabular}{ccc}
\hline $\begin{array}{c}\text { Composición } \\
\text { química }\end{array}$ & $\%$ & Método utilizado \\
\hline Silicio (Si) & 55.82 & Espectroscopía \\
Aluminio (Al) & 11.73 & de energía \\
Hierro (Fe) & 9.93 & dispersiva (EDS) \\
Calcio (Ca) & 6.19 & \\
Magnesio (Mg) & 4.51 & \\
Potasio (K) & 9.53 & \\
Sodio (Na) & 2.27 & \\
\hline
\end{tabular}

Fuente: Ceniza de bagazo de caña de azúcar en la resistencia a la compresión del concreto, Universidad César Vallejo de Perú.

Tabla 4. Composición química inorgánica de las cenizas de bagazo de caña de azúcar (CBCA) expresada como óxidos.

\begin{tabular}{lcc}
\hline \multicolumn{1}{c}{ Composición química } & $\%$ & Método utilizado \\
\hline Dióxido de Silicio (SiO2) & 64.04 & \\
Trióxido de Aluminio & 11.89 & \\
(Al2O3) & 7.61 & $\begin{array}{c}\text { Espectroscopía de } \\
\text { energía dispersiva } \\
\text { (EDS)* }\end{array}$ \\
Trióxido de Hierro & 4.65 & \\
(Fe2O3) & 4.01 & \\
Óxido de Calcio ( $\mathrm{CaO})$ & 6.16 & \\
Óxido de Magnesio (MgO) & & \\
\hline
\end{tabular}

Fuente: Ceniza de bagazo de caña de azúcar en la resistencia a la compresión del concreto, Universidad César Vallejo de Perú

En la tabla 5 se puede observar el asentamiento del concreto en estado fresco.
Tabla 5. Asentamiento del concreto en estado fresco

\begin{tabular}{ccc}
\hline Mezcla & $\begin{array}{c}\text { Slump máximo } \\
\text { (pulg.) }\end{array}$ & \% variación \\
\hline $\mathrm{C}^{\circ} \mathrm{E}$ & 3 & $0 \%$ \\
$\mathrm{C}^{\circ}+20 \%$ & 3.24 & $8 \%$ \\
$\mathrm{C}^{\circ}+40 \%$ & 3.17 & $5.70 \%$ \\
\hline
\end{tabular}

Fuente: Ceniza de bagazo de caña de azúcar en la resistencia a la compresión del concreto, Universidad César Vallejo de Perú.

De la tabla 6 se puede observar que el asentamiento en las mezclas de concreto con 20 y $40 \%$ de CBCA tuvieron un incremento $8 \%$ y $5.7 \%$, respecto al concreto estándar.

La consistencia obtenida de todas las mezclas producidas y ensayadas fueron las deseadas, no se obtuvo diferencias importantes a pesar de los diferentes porcentajes del CBCA, siendo este un reemplazo parcial del cemento dentro del concreto aunque en otros estudios realizados [18] se demostró que se obtienen asentamientos por el orden del $33.33 \%$ al usar $40 \%$ de la ceniza en la mezcla de concreto como sustituyente del concreto.

Tabla 6. Resistencia a la compresión promedio del concreto endurecido a 7 y 28 días de curado

\begin{tabular}{lcccc}
\hline \multirow{2}{*}{ Mezcla } & \multicolumn{4}{c}{$\mathrm{f}^{\prime} \mathbf{c}(\mathbf{k g} / \mathbf{c m} 2)$} \\
\cline { 2 - 5 } & $\mathbf{7}$ dias & $\begin{array}{c}\% \\
\text { Alcanzado }\end{array}$ & 28 dias & $\begin{array}{c}\% \\
\text { Alcanzado }\end{array}$ \\
\hline $\mathrm{C}^{\circ} \mathrm{E}$ & 144.25 & $68.69 \%$ & 212.75 & $101.31 \%$ \\
$\mathrm{C}^{\circ}+20 \%$ & 140 & $66.67 \%$ & 162.75 & $77.50 \%$ \\
$\mathrm{C}^{\circ}+40 \%$ & 117.75 & $56.07 \%$ & 162.5 & $77.38 \%$ \\
\hline
\end{tabular}

Fuente: Ceniza de bagazo de caña de azúcar en la resistencia a la compresión del concreto, Universidad César Vallejo de Perú.

En las figuras 3 y 4 se puede observar la resistencia a la compresión del concreto según el porcentaje de ceniza de bagazo de caña de azúcar. 


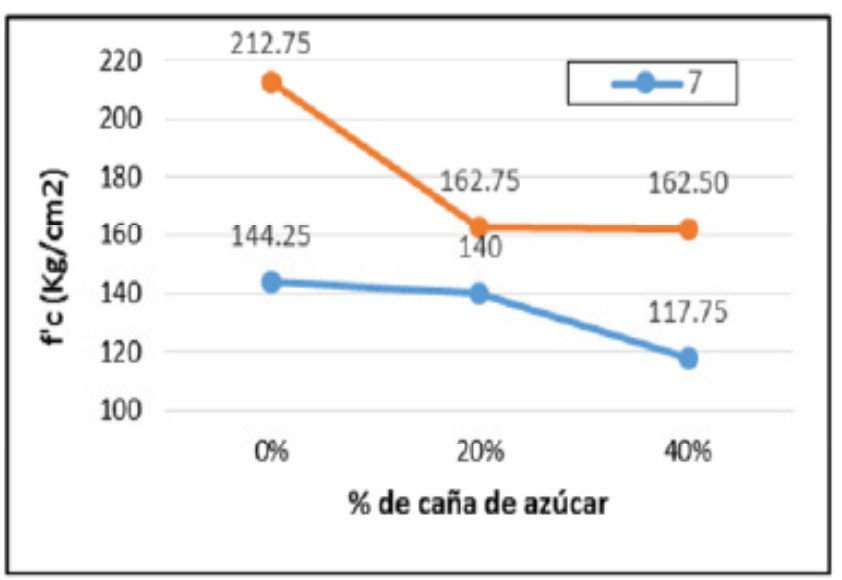

Figura 3. Resistencia a la compresión del concreto según porcentaje de ceniza de bagazo de caña de azúcar

Fuente: Ceniza de bagazo de caña de azúcar en la resistencia a la compresión del concreto, Universidad César Vallejo de Perú.

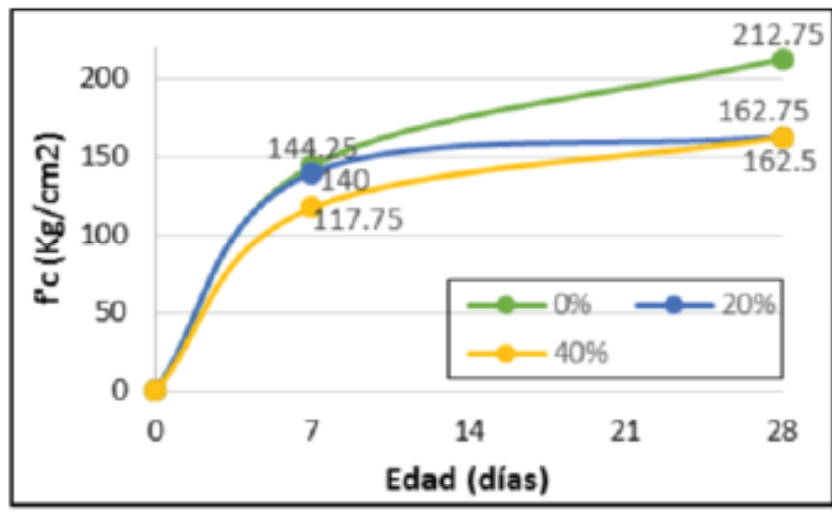

Figura 4. Resistencia a la compresión del concreto según tiempo de curado Fuente: Ceniza de bagazo de caña de azúcar en la resistencia a la compresión del concreto, Universidad César Vallejo de Perú.

Ceniza de bagazo de caña como aditivo al cemento Portland para la fabricación de elementos de construcción, Universidad Nacional de Colombia (Sede Palmira, Valle del Cauca).

El Valle del Cauca, por sus condiciones ambientales, tiene alta presencia de cultivos azucareros. Un residuo del procesamiento de la caña, poco explorado es la ceniza del bagazo (CBC), objetivo del presente estudio. El bagazo de caña de azúcar una vez es utilizado en las calderas industriales, da lugar a la CBC, este material al igual que los residuos del carbón o cenizas volantes es considerado un material con carácter puzolánico por algunos investigadores. Se estudiaron tres cenizas procedentes de ingenios azucareros del Valle del Cauca, por medio de composición química, difracción de rayos $\mathrm{X}$ y actividad puzolánica. Se encontró que las $\mathrm{CBC}$ analizadas contienen Sílice (SiO2) y Alúmina (Al2O3), en diferentes proporciones, y que poseen amorficidad en su estructura, lo cual favorece la reactividad con cemento.

Las muestras fueron tratadas térmicamente debido al alto contenido de inquemados, y se les determinó la actividad puzolánica, encontrándose índices hasta del 97\%, cumpliendo a cabalidad con la norma ASTM C618.

Tabla 7. Características físicas y químicas de las cenizas y el cemento.

\begin{tabular}{lcccc}
\hline \multicolumn{1}{c}{ Características (\%) } & CBC1 & CBC2 & CBC3 & Cemento \\
\hline $\mathrm{SiO}_{2}$ & 58,6 & 76,4 & 63,2 & 24,3 \\
$\mathrm{Al}_{2} \mathrm{O}_{3}$ & 11,8 & 5,8 & 8,5 & 4,3 \\
$\mathrm{Fe}_{2} \mathrm{O}_{3}$ & 5,8 & 4,5 & 6,4 & 3,0 \\
$\mathrm{CaO}$ & 3,0 & 3,3 & 3,9 & 58,8 \\
$\mathrm{MgO}$ & 2,2 & 2,3 & 4,3 & 1,4 \\
$\mathrm{~K}_{2} \mathrm{O}$ & 2,0 & 4,2 & 7,3 & 0,7 \\
$\mathrm{Na}_{2} \mathrm{O}$ & 1,3 & 1,2 & 1,1 & 0,8 \\
Pérdidas por ignición & 10,0 & 2,0 & 11,0 & 4,0 \\
Tamaño de partícula (um) & 38,7 & 79,8 & 41,5 & 16,0 \\
\hline
\end{tabular}

Fuente: Ceniza de bagazo de caña como aditivo al cemento Portland para la fabricación de elementos de construcción, Universidad Nacional de Colombia (Sede Palmira, Valle del Cauca).

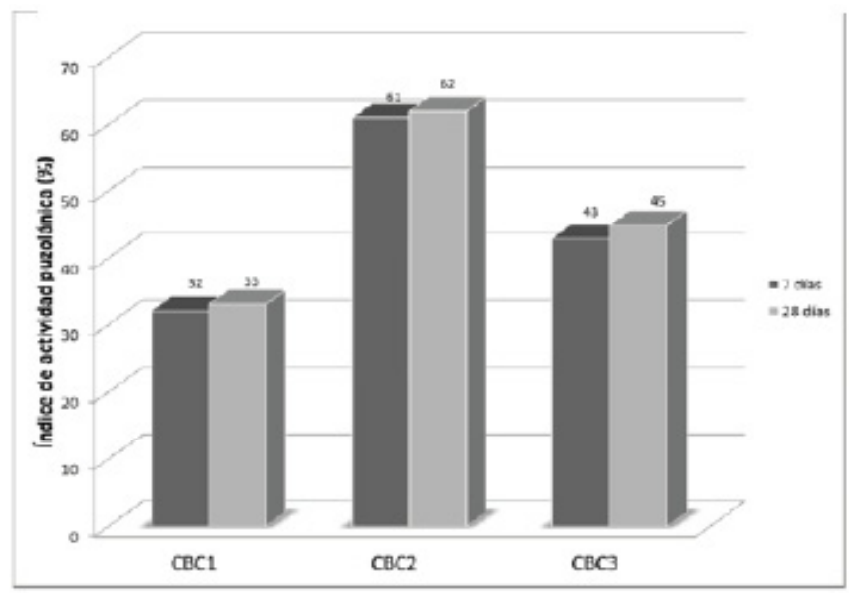

Figura 5. Índice de actividad puzolánica 
Análisis comparativo de resultados en el uso de la ceniza de bagazo de caña de azúcar como material sustituyente del cemento portland en el concreto

\section{6}

Figura 6. Índice de actividad puzolánica para las cenizas tratadas térmicamente.

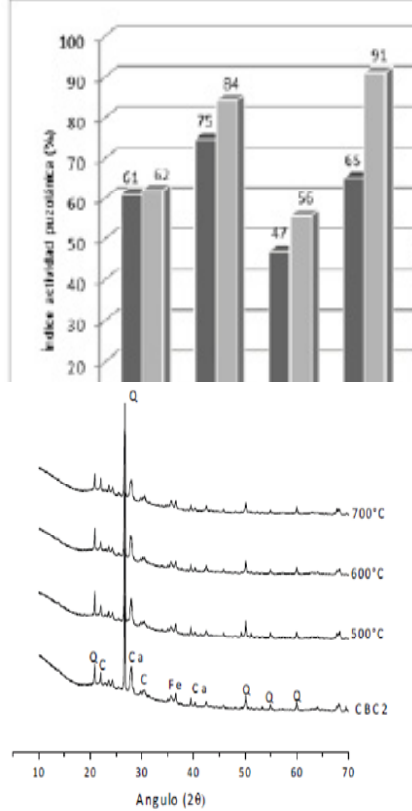

a) $\mathrm{CBC} 2$

Figura 7. Difractogramas de Rayos $X$ para las cenizas tratadas térmicamente.

Así mismo, en los resultados obtenidos en la segunda investigación analizada y al igual que en la anterior, a las cenizas tomadas como muestras para el estudio se les determinó su índice de actividad puzolánica (IAP) a través de la resistencia a la compresión, según las normas ASTM C311 y C642 las cuales consisten en la preparación de morteros con una adición porcentual con respecto a la cantidad de cemento. En la figura 8 se puede observar que las muestras al ser tratadas térmicamente presentan cantidades de cuarzo, lo cual se le atribuye a la arena en la caña al momento de ser cosechada.

\section{Conclusiones}

De acuerdo con las investigaciones analizadas se puede concluir que no se recomienda el uso de la Ceniza de Bagazo de caña en proporciones de $20 \%$ y $40 \%$ como sustituyente del cemento para la fabricación de mezcla de concreto estructural debido a su disminuida resistencia a la compresión. También, que las cenizas tomadas como muestras para las investigaciones requirieron de un tratamiento térmico, con una temperatura ideal de $700^{\circ} \mathrm{C}$, para mejorar sus propiedades puzolánicas y su reactividad frente al cemento.

En ambos estudios se destaca la reutilización de un residuo industrial, ya que su aplicación lograría disminuir el consumo de cemento, dándose así un efecto ambiental positivo, pues la producción de este último es altamente contaminante. Se sugieren hacer estudios adicionales de resistencias mecánicas y de durabilidad en materiales cementicios adicionados con este residuo.

\section{Referencias}

[1] J.I.E. Garcia, "GeoCities", 2002. [En línea]. Disponible en: http://www.geocities.ws/ rosenberg_rd/tema2-3-7.pdf

[2] C. Mansaneira, N. Schwantes-Cezario, G. F. Barreto-Sandoval y B. Martins-Toralles, "Ceniza de bagazo de caña de azúcar como material puzolánico", $\boldsymbol{D Y N A}$, vol. 84, no. 201, pp. 163-171, June, 2017

[3] C.E.G. Escandón, D.V.V. Velasco, C.M. Lopez, J. T. Ágredo y L.O.G. Salcedo, "Ceniza de bagazo de caña como aditivo al cemento Portland para la Fabricacion de Elementos de Construcción", Palmira, Valle del Cauca, 2012.

[4] P. Camargo Macedo, A.M. Pereira, J.L Akasaki, C.F. Fioriti, J. Payá y J.L. Pinheiro, "Rendimiento de morteros producidos con la incorporación de ceniza de bagazo de caña de azúcar", Revista ingeniería de construcción, vol. 29, no. 2, pp. 187-199, 2014

[5] C.E. Giraldo Escandón, D.V. Vidal Velasco, C. Martínez López y J. Torres Agredo, "Ceniza de bagazo de caña como aditivo al cemento Portland para la fabricación de elementos de construcción", Acta Agronómica, vol. 61, no. 


\section{5,2012}

[6] Conab, "Ministério da Agricultura, Pecuária e Abastecimento", 15 Septiembre 2016. [En línea]. Disponible en: https:/www.gov.br/agricultura/ assuntos/sustentabilidade/agroenergia/ arquivos-termoeletrica-com-a-queima-dobagaco-de-cana-deacucar/termoeletrica-coma-queima-do-bagaco-de-cana-de-acucarnobrasil-safra-2009-2010.pdf

[7] FIESP/CIESP, "Federação das indústrias do estado de São Paulo/centro das indústrias do estado de São Paulo", 15 Septiembre 2016. [En línea]. Disponible en: http://www.fiesp.com.br/ arquivodownload/?id=4505

[8] E. M.R. Fairbairna, B. B. Americano, et al., "Cement replacement by sugar cane bagasse ash: $\mathrm{CO} 2$ emissions reduction and potential for carbon credits", Journal of Environmental Management, vol. 91, no. 9, pp. 1864-1871, 2010

[9] MANUELITA S.A., Así producimos azúcar alta pureza: Diagrama del proceso de elaboración de azúcar y sus derivados, Valle del Cauca, 2010.

[10] V.C. Gálvez, "Pacific Credit Rating", 2011. [En línea]. Disponible en: Available: http://www.ratingspcr.com/boletin/main. php?K=3931\&id=122

[11] J.I. Escalante, "Materiales alternativos al cemento Portland. Avance y Perspectiva", Periódica, vol. 21, pp. 79-88, 2002

[12] J. Payá, J. Monzó, M.V. Borrachero, L.D. Pinzón y L. Ordóñez, Sugarcane Bagasse ash (SCBA) :Studies on its properties for reusing in concrete production, Journal of chemical Technology and Biotechnology, vol. 77, no. 3, pp 321-325, 2002
[13] G.C. Cordeiro, et al., "Pozzolanic activity and filler effect of sugar cane bagasse ash in Portland cement and lime mortars" Cement and Concrete Composites, vol. 30, no. 5, pp. 410-418, May 2008,

[14] D.V. Vidal, J. Torres y L.O. González, “Ceniza de bagazo de caña para elaboración de materiales de construcción: Estudio preliminar", Revista de Fisica, p. 14, 2014.

[15] J. Herrera, "Wordpress", Noviembre 2008. [En línea]. Disponible en: https://juanherrera. files.wordpress.com/2008/11/investigacioncuantitativa.pdf.

[16] INCAUCA, "INCAUCA", Mayo 2016. [En línea]. Disponible en:https://www.incauca.com/ wp-content/uploads/2016/05/PROCESOS ESPANOL.pdf

[17] M. Frias, E. Villar- Cociña y E. ValenciaMoraes, "Characterization of sugar cane straw waste as pozzolanic material for construction: Calcining temperature and kinetic parameters", Waste Management, vol. 27, no. 4, pp. 533-538, 2007

[18] A. Garcia-Rodriguez, A. Marin-Cardenas, N. Herrera-Coello, I. Diaz-Rodriguez, y L. Marrero-Suarez, "Produccion de biomasa proteica a partir de jugo de caña", Respuestas, vol. 4, no. 1, pp. 32-34, jun. 2016

[19] Y.D.J. Cabrera, "Evaluación del efecto de la adición de cenizas volantes producto de la incineración del bagazo de caña de azúcar en mezclas de concreto de bajas a medias resistencias como sustitución parcial del cemento", Universidad Central de Venezuela, 2010 